\title{
Beraten und steuern in der Sozialen Arbeit
}

\section{Zwei Seiten einer Medaille}

\author{
Ruth Remmel-Faßbender
}

\begin{abstract}
Das professionelle Begleiten und Unterstützen von Menschen in schwierigen Lebenssituationen verlangte schon immer das Steuern von Hilfen im Sinne von Verselbstständigung und Lösungsorientierung sowie des Überprüfens vereinbarter Ziele. Seit einigen Jabren kommen als Aufgaben hinzu die Prüfung der Wirksamkeit der Hilfen und die Kontrolle des erforderlichen Aufwands.
\end{abstract}

Beratung und Management, Case Management und Systemsteuerung, Counceling und Fallsteuerung, Fallmanagement und Betreuungsunterstützung - immer häufiger treten diese Begriffskombinationen in der Fachdebatte um die Handlungsorientierung Sozialer Arbeit auf. Je nach inhaltlicher Positionierung entweder unter integrativer Perspektive oder in einer kritischen, unvereinbaren Gegenüberstellung. Was ist der aktuelle Hintergrund dieses Diskurses über das Verhältnis von Kompetenzen, Standards und Prinzipien von Beratung und politisch, organisatorischen Steuerungsvorgaben?

Seit Beginn der Diskussion über die Ökonomisierung Sozialer Arbeit wird die Auseinandersetzung zwischen professioneller »helfender « Beziehungsgestaltung und strukturell bedingten, als »berufsfremd « empfundenen (z. B. die Beachtung beschränkter Ressourcen) Rollenanforderungen mit vielerlei Facetten geführt. Für die schwierige Frage, wie Beratungskonzepte im Kontext veränderter Bedingungen den Zielen unterstützungsbedürftiger Menschen, aber auch den Zielen veränderter Organisations- und Finanzierungs-

Prof. Ruth Remmel-Faßbender lehrt Methoden in der Sozialen Arbeit an der Katholischen Fachhochschule Mainz. Sie ist Diplom-Pädagogin, DiplomSozialarbeiterin, Case-ManagementAusbilderin (DGCC) und Supervisorin (DGSv).

E-Mail remmel-fassbender@kfh-mainz.de strukturen gerecht werden können, werde ich einige Leitgedanken für die konkrete Handlungsebene skizzieren.

Beratung als Grundkompetenz und zentrale Handlungsweise Sozialer Arbeit sowie ihre spezifische Form unterstützender Interaktion mit dem Ziel der Aktivierung von Menschen zu ihrer Problemlösungs- und Selbststeuerungsfähigkeit ist unstrittig Bestandteil ganzheitlicher Handlungskompetenz. Gerade weniger privilegierte Menschen mit Vielfachbelastungen und komplexen, lang andauernden Problemlagen benötigen Beratung, Begleitung und Zeit und Raum, um Kompetenz und Selbstwert zur eigenen, (mit-) verantworteten Lösung entwickeln zu können. Beratung mag ein schillernder Begriff sein, aber Schlüsselqualifikationen und Anforderung an Mitarbeiterinnen und Mitarbeiter sind für die Soziale Arbeit hinreichend fachlich beschrieben.

Beratung in der Sozialen Arbeit geschieht unter systematischer Einbeziehung der sozialen Umwelt der Klienten, ihrer personalen und sozialen Ressourcen, der Nutzung der sozialen Hilfesysteme und der Gestaltung gesellschaftlicher Kontexte. Das im Orientierungsrahmen der Deutschen Gesellschaft für Beratung/German Association for Counseling (DGfB) erarbeitete Qualifikationsprofil ist gekennzeichnet von einer hohen Ressourcen- und Lebensweltorientierung jenseits klinisch-therapeutischer Traditionen (vgl. Internet http://www. dachverband-beratung.de, Stand 10. 12. 2006, Wendt 2006a, S. 15 f.)

Beratung findet in unterschiedlichen Kontexten und Settings statt. Beratung und Kontrolle (doppeltes Mandat), Beratung im Pflichtkontext, in konfliktbeladenen schwierigen Lebenssituationen und mit unmotivierten Klientinnen und Klienten sind nur einige Beispiele, die die Komplexität und die hohen Anforderungen an Beratungsqualifikationen kennzeichnen.

Erfordert das Begleiten und Unterstützen von Menschen in schwierigen Lebenssituationen immer auch das Steuern von Hilfen im Sinne des Lenkens auf Verselbstständigung und Lösungsorientierung hin, des Überprüfens vereinbarter Ziele, so kommen seit einigen Jahren die Überprüfung von Ertrag (Wirksamkeit) und erforderlichem (wirtschaftlichem) Aufwand als Aufgaben hinzu.

\section{Der Legitimationsdruck für die Soziale Arbeit verschärft sich}

Die Forderung nach der Steuerung sozialer Dienstleistungen begleitet seit über 20 Jahren die Umstrukturierungs- und Anpassungsprozesse in sozialen Arbeitsfeldern. Der Wandel sozialer Verhältnisse hat eine kontinuierliche Neustrukturierung Sozialer Arbeit in vielen Bereichen zur Folge, weil herkömmliche Hilfen nicht mehr greifen und sozialstaatliche Leistungen nicht immer ausreichend sind. Der Legitimationsdruck Sozialer Arbeit durch leere Haushaltskassen, aber auch durch »Markterfordernisse ", verschärft sich.

Die Verknüpfung der Hilfemaßnahmen mit dem Anspruch nach administrativer und ökonomischer Steuerung erfolgt gezielt in den öffentlichen Verwaltungen seit Beginn der 1990er Jahre aufgrund der »Neuen Steuerungsmodelle « der Kommunalen Gemeinschaftsstelle (KGSt). Im Gefolge hat das Qualitätsmanagement Einzug in die sozialen Dienstleistungen gehalten. Aus dem politischen Kontext von »New Public Management " (Reduzierung der staatlichen Verantwortung für bislang gesetzlich verankerte Wohlfahrtsleistungen) und »aktivierender Staat « (Leistungen werden erbracht nach dem Prinzip »Fördern und Fordern «) resultieren weitere Reformbestrebungen, die die Steuerungsfrage noch stärker in den Vordergrund stellen.

Die neueren Sozialgesetzbücher und ihre Ergänzungen (z. B. in der Jugendhilfe, Beschäftigungsförderung, Rehabilitation, Integrierten Versorgung) schaffen auf der Strukturebene die Rahmenbedingungen für die Bedarfsplanung, um auf 
der Einzelfallebene dann die Leistungen entsprechend zu planen und zu erbringen. Die Steuerungsfunktion ist sozialpolitisch intendiert und soziale Unterstützungsmaßnahmen sind von der Diskussion um Steuerung nicht mehr abzukoppeln. Der Bedarf in öffentlichen Verwaltungen und bei freien Trägern nach strategischen Steuerungskonzepten nimmt zu.

Die Fachkräfte auf der operativen Ebene sind heute überwiegend abhängig von der wirtschaftlichen Steuerung (Controlling) ihres Trägers. Obwohl viele Dienste bedarfsgerechte, koordinierte, transparente Hilfen nicht nur als effektiver und damit auch effizienter ansehen, befürchten viele Fachkräfte dennoch den Verlust sozialarbeiterischer Fachlichkeit, wenn der Kosteneinsparungseffekt als Entscheidungskriterium dominiert.

Werden dagegen beispielsweise nur kurzfristige Angebote gemacht oder gar notwendige Hilfen aus Kostengründen verworfen, kann dies betriebswirtschaftlich sinnvoll sein, aber zu hohen volkswirtschaftlichen Folgekosten führen. Das ist Allgemeinwissen und führt trotzdem nicht in ausreichendem Maße zur bedarfsgerechten nutzerorientierten Vernetzung auf der Versorgungsebene. (1) Fallübergreifende sinnvolle Steuerungsverfahren werden trotz aller gesetzlichen Vorgaben und Plausibilitätseinsichten letztlich nicht tiefgreifend und konsequent in der Versorgungsstruktur implementiert.

Bei der Recherche nach Ergebnissen wirkungsorientierter Steuerung in sozialen Arbeitsfeldern wird man allerdings kaum fündig. Es gibt wenig empirische
Belege, ob die gezielte Lenkung von Leistungen für die unterschiedlichen Ebenen die erwarteten Resultate erbracht hat (Schröder/Kettiger 2001).

Bedarfs- und angebotsorientierte Steuerung ist zwar attraktiv für Soziale Arbeit, weil sie großes Interesse daran hat, fachlich positive Wirkungen zu erzielen. Mit möglichen »Wirkungen « öffnet sich jedoch ein hoch komplexes Feld, denn erwartete Wirkungen von Klienten, Professionellen, Geldgebern, dem Gesetzgeber können je nach (auch gesellschaftlich definiertem) Bedarf und der Festlegung sozialpolitischer Ziele höchst unterschiedlich sein (wie die Diskussion um »Hartz $\mathrm{IV}$ « und die Folgen eindrücklich zeigt).

Veränderungen bei Menschen erfolgen nicht linear und hängen von vielen anderen Beeinflussungsfaktoren als der professionellen Hilfe ab. Sie wirken oft erst längerfristig oder im Verborgenen. Ob und unter welchen Zielvorgaben es gelingt, Menschen zur Selbstsorge, letztlich zur Selbststeuerung ihrer Angelegenheiten zu befähigen, muss in fachlichem Interesse stärker systematisch evaluiert werden (vgl. Kasten Seite 88: »Wie beraten? «). (2)

\section{Wohin das Schiff steuert}

Die Fokussierung auf eine bedarfsorientierte Einzelfallunterstützung setzt sich durch. Sie führt weg von der umfassenden, zeitlich unbegrenzten Beratung hin zu einer zeitlich begrenzten, lösungsorientierten, aktivierenden Beratung, die an den Be- darfen der Menschen ansetzt: Gesundheit, Bildung, Sprachkenntnisse, berufliche Orientierung, Familie, andere Unterstützungspotenziale. Hinzu kommt eine zunehmende Öffnung von sozialen Diensten und Einrichtungen hin zum Sozialraum.

Die konsequente Vernetzung zu einer interdisziplinären, fallübergreifenden Zusammenarbeit nicht nur mit Beratungsdiensten und sozialen Einrichtungen, sondern auch mit anderen beteiligten Fachkräften wie Controller und Qualitätsmanager, also planenden und disponierenden Funktionen, bedeutet ein Umdenken. Nach dem ersten Zusammenprall unterschiedlicher Werturteile und Kulturen (berufsspezifische Sichtweisen, internalisierte Rollenzuschreibungen, alte Feindbilder) resultiert daraus eine qualitativ andere, von Verständnis getragene Wahrnehmung beteiligter Dienste und eine gegenseitige fachliche Ressourcennutzung.

Ein in seinen Grundprinzipien (4) und seiner Spezifik umgesetztes Case Management in Handlungsfeldern der Sozialen Arbeit bleibt trotz oder wegen seiner ökonomischen Ausrichtung im Verfahren eine personenbezogene, auf Kontinuität in der Begleitung ausgerichtete Dienstleistung, die die vielfältigen Leistungen steuert.

Der Weg Sozialer Arbeit zu einem stärker wirtschaftlich orientierten Denken in Bezug auf Leistung, Effizienz und Effektivität schreitet fort. Gleichzeitig verschärfen sich Problemsituationen und der Handlungsdruck (siehe die zahlreichen Meldungen der letzten Monate über gefährdete Kinder in überaus problembehafteten $\mathrm{Fa}$ -

\section{Literatur}

Deutsche Gesellschaft für Beratung/German Association for Counseling (DGfB). http://www.dachverband-beratung.de/Dokumente/Beratung.pdf. Stand: 10.12.2006.

Gissel-Palkovich, Ingrid (2006): Case Management - ein Handlungskonzept Sozialer Arbeit? In: Sozialmagazin, 31. Jg., Heft 2.

Herrmann-Woitas, Eva (2000): Integrierte Rehabilitations- und Behandlungsplanung im psychiatrischen Wohnverbund. Eine Prozess- und Ergebnisevaluation. Heidelberg.

Klug, Wolfgang (2006): »Fallmanagement « Eine Konstruktion des SGB II. In: Sozialmagazin, 31. Jg. Heft 2, 20.

Löcherbach, Peter/Klug, Wolfgang/
Remmel-Faßbender, Ruth/Wendt, Wolf Rainer (2005): Case Management. Fallund Systemsteuerung in der Sozialen Arbeit. München: Reinhardt. 3. Aufl.

Neuffer, Manfred (2006): Case Management am Scheideweg. Ein Beitrag aus der Sicht der Sozialen Arbeit. In: standpunkt: sozial. Heft 2/2006.

Remmel-Faßbender, Ruth (2006a): Handlungskompetenzen in der Fallsteuerung. Anforderung an Weiterbildungsinhalte. In: Zeitschrift Case Management 1/2006.

Remmel-Faßbender, Ruth (2006b): Case Management in der Jugendhilfe. Der Versuch einer aktuellen Bestandsaufnahme. In: Wendt, W. R./Löcherbach, P.: Case Management in der Entwicklung Stand und Perspektiven in der
Praxis. Heidelberg: Economia.

Schröder, Jan/Kettiger, Daniel (2001): Wirkungsorientierte Steuerung in der Sozialen Arbeit. Ergebnisse einer internationalen Recherche in den USA, den Niederlanden und der Schweiz. Im Auftrag des Bundesministeriums für Familie, Senioren, Frauen und Jugend. Kohlhammer. Wendt, Wolf-Rainer (2006a): State of the art: Das entwickelte Case Management. In Wendt/Löcherbach (Hg.): Case Management in der Entwicklung. Stand und Perspektiven in der Praxis.

Wendt, Wolf-Rainer (2006b): Ökonomie im Case Management: Allokation auf den Ebenen der Versorgung und fallweise Transaktion. In: Zeitschrift Case Management 2/2006. 
milien). Die sozialen Dienstleistungen müssen sich daher weiter mit der Entwicklung und Sicherstellung von Standards offensiv in die fachliche Auseinandersetzung um Steuerungskonzepte begeben, damit am tatsächlichen Bedarf angemessene Unterstützungsprozesse gewährt werden.

Der »Luxus « dieser beratenden Unterstützung zahlt sich dann vielfach aus, denn ein im erfolgreichen Zusammenspiel zwischen Sozialarbeiteterin und Sozialarbeiter sowie Klientin und Klient gefördertes Selbstbewusstsein der Betroffenen ist im Sinne des Urprinzips »Hilfe zur Selbsthilfe « und des Systems fruchtbar und ertragreich. Wenn durch Vertrauen in einer sozialen Beratungs- und Arbeitsbeziehung Fordern und Fördern nach dem Maß der individuellen Möglichkeiten eines Menschen zusammen kommen, dann sind Beratung und Steuerung zwei erforderliche, komplementäre Seiten einer Medaille.

\section{Anmerkungen}

(1) Vgl. hierzu ausführlich die Ausführungen von Wendt 2006b zum ökonomischen Zusammenhang und sozialwirtschaftlichen Nutzen der Steuerung von Hilfen auf der Makroebene (Versorgungsleistungen des Staates), der Mesoebene (Erbringung von Leistung auf der Steuerungsebene der Organisation) und der Mikroebene (Verknüpfung der erforderlichen Ver- sorgungsleistung mit der Selbstsorge von Nutzerinnen und Nutzern in der humandienstlichen Versorgung).

(2) Zu Konsequenzen möglicher Machtasymmetrien bei steuerungspolitischen Leistungsvereinbarungen und der Einengung fachlicher Ermessens- und Handlungsspielräumen im Rahmen Sozialer Arbeit sei auf Klug 2006; Neuffer 2006 verwiesen.

(3) Zur Problematik des Fallmanagements im SGB II, dass Steuerung im Kontext der Berufseingliederung mit Sanktionen verknüpft wird, siehe Siglinde Bohrke-Petrovic in diesem Heft

(4) Vgl. die »Leitprinzipien DGS, DBSH DBfK zum Case Management " (Internet http://www.dgcc.de).

\section{Wie beraten?}

Handlungsbezogene Steuerung geschieht auf den Ebenen der direkten Einzelfallsteuerung und der Systemsteuerung. Steuerung auf der Einzelfallebene (Mikroebene) geschieht beispielsweise durch den Zugang zur Beratung: über Clearingstellen oder die »Verteilung « nach Fallgruppen, über Bedarfsfeststellung und Zielfindung, Hilfe- oder Förderplanung, kontinuierliche Überprüfung der Maßnahmen und eine abschließende, gemeinsame Auswertung. Sie ist bestenfalls geknüpft an ein systematisches, dokumentiertes Ablaufverfahren in klar definierbaren Schritten. Voraussetzung für das Steuern auf der Einzelfallebene ist: Sowohl in Organisationen als auch im regionalen Versorgungsnetz müssen Rahmenbedingungen für Abläufe (Pfade), Standards, konkrete Handlungsanweisungen (Manuale) erarbeitet worden sein.

Case Management gilt in diesem Zusammenhang als das Verfahren der Wahl. Die aktuellen Entwicklungen führten schnell zur Übernahme des Konzepts im Sozial- und Gesundheitswesen, das für sich als integratives ganzheitliches, interprofessionelles Konzept fachlich und ökonomisch sinnvolles Handeln beansprucht.

Nach Wendt geht es im Steuern des Care- und Case Managements »ökonomisch darum, den Haushalt einer Versorgungsgemeinschaft (einer Kommune, einer Arbeitsverwaltung, einer Versicherung) in transparenter Weise mit den Haushaltsentscheidungen von zu versorgenden Menschen und den für sie dienstlich Handelnden zu verbinden " (Wendt 2006b, S, 71). Case Management als kooperativer Prozess erhebt, plant, implementiert, koordiniert, überwacht und evaluiert den individuellen Unterstützungsbedarf. Beratung im Case Management umfasst die doppelte Perspektive zu Klienten und zum Versorgungssystem und geht damit über den persönlichen Interaktionsprozess im förderlichen Sinn hinaus. Systemsteuerung und Anliegen der Nutzerinnen und Nutzer müssen sich gegenseitig durchdringen und umgekehrt (Gissel-Palkovich 2006; Wendt 2006a, S. 7).

Durch die Festlegung fachlicher Standards und systematischer Verfahren (der Hilfeplanung nach $\mathbb{} 36$ SGB VIII, dem Integrierten Behandlungs- und Rehabilitationsplan für psychisch kranke Menschen, Integrationsförderplänen usw.) wurden In- strumente einer einzelfallbezogenen Steuerung geschaffen, die eine aktive Einbeziehung unterstützungsbedürftiger Menschen ermöglichen. Case Management auf der Einzelfallebene erfolgt damit in kleinen Schritten und am individuellen Bedarf ausgerichtet.

Vielerorts entwickelt wurden praktikable, aus der Profession stammende Verfahren zur Ermittlung des tatsächlichen Bedarfs, klein dimensionierte ziel- und zeitorientierte Maßnahmen, mögliche Indikatoren und Messverfahren zur Verlaufskontrolle und abschließender Evaluation. Im Verständnis einer passgenauen, personenbezogenen Leistung führen diese bei fachlichem Einsatz zur höheren Zufriedenheit bei den Nutzern und den Fachkräften (Woitas 2000). Professioneller Einsatz meint die empathische Handhabung und Interpretation durch im Verfahren qualifizierte Fachkräfte - und nicht die verwaltungsmäßige, technische Abarbeitung von Assessmentbögen, die dann von beiden Seiten als lästige, unnütze Zusatzarbeit empfunden wird.

Grundsätzlich kann sich Beratung in ein Case Management einordnen, muss es aber dann nicht, wenn Menschen über genügend Ressourcen und Kräfte verfügen, um in Koproduktion mit der Case Managerin oder dem Case Manager ihre komplexen Anliegen weitgehend selbst zu steuern. In zahlreichen Handlungsfeldern der Sozialen Arbeit allerdings erfordert Case Management eine Beratungsqualifikation für kommunikative Aushandlungsprozesse sowie eine dialogische Steuerung zur Begleitung von Menschen in schwierigen Ausgangssituationen, die nicht oder vorübergehend nicht in der Lage sind, ihre vielschichtigen Angelegenheiten selbstverantwortlich zu gestalten.

Wer beispielsweise im SGB II (3), beim Persönlichen Budget oder in der gesetzlichen Betreuung "auf gleicher Augenhöhe « Vereinbarungen schließen und seine Interessen einbringen will, muss auch in einem möglichen Pflichtkontext in seinen »Sinnkonstruktionen ", seinem Alltagsverständnis, seinen unterschiedlichen Problembereichen einen verantwortungsvollen Umgang erfahren, sonst wird er einen zusätzlichen Berater zum Case Manager benötigen, der für ihn anwaltschaftlich eintritt. 